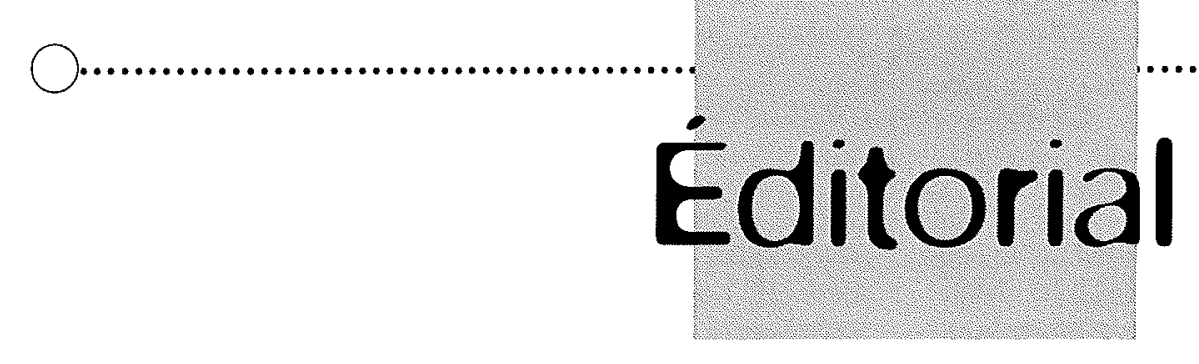

\title{
Dormir, rêver peut-être
}

\author{
Claude MIOLLAN*, Thierry BISSON**
}

* Professeur de Psychologie Clinique et Pathologique. Université de NiceSophia Antipolis, 06560 Sophia Antipolis, France. miollan.claude@free.ft * Maitre de Conférence en Psychologie clinique bissonthierry@ wanadooff

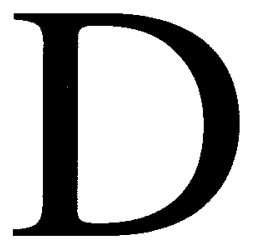

ormir, rêver peut-être, c'est ce que nous espérons tous vivre au moment de nous mettre au lit. S'endormir simplement n'est pas chose si simple. Nous esquisserons ici une réflexion sur cette zone de l'endormissement comme travail de la dynamique, de l'alternance de l'illusion-désillusion, et nous en soulignerons quelques enjeux.

La psychopathologie des troubles du sommeil (endormissement et réveil) comme presque toujours en psychologie clinique est à la source de notre savoir sur la question.

Raconter ses rêves est devenu un stéréotype de notre culture sous influence psychanalytique. Nous remarquerons pourtant que l'on obtient plus rarement le récit de ces fantaisies, de ces mouvements psychiques qui occupent le dormeur potentiel ; peut-être à cause de l'état modifié de la conscience à ce moment-là, mais pas seulement. Certains patients en analyse avouent cette difficulté à nous confier ce dernier lambeau de leur intimité, d'autres le vivent même comme l'envers de leurs rêves, de ces rêves qu'ils nous racontent. Nous aurions sans doute la même difficulté à obtenir le récit des mouvements internes des spectateurs après un spectacle. Comme de bons critiques, ils théoriseraient doctement sur la mise en scène, l'interprétation et le reste. Pourtant le plaisir est plus évident si l'on accepte de se laisser surprendre. Mais se laisse-t-on surprendre par le sommeil en dehors des cas d'épuisement ? Se laisse-ton surprendre par le sommeil, surtout si l'on pense qu'il s'agit de se mettre dans un état de mort apparente.

Les noctambules, dont la littérature et le cinéma ont souvent décrit les errements nocturnes, nous montrent qu'il faut beaucoup cheminer avant qu'au bout du voyage la conscience ne cède. S'endormir est un cheminement, mais le chemin n'est pas vraiment balisé. Plus qu'un chemin, c'est un passage, une sorte de couloir secret, un escalier dérobé que l'on emprunte toujours seul. Chacun ritualise, parfois à son insu, ce moment de transition entre veille et sommeil, chacun a sa méthode. Eclitico dans l'opéra «Le monde de la lune» (livret de Goldoni) invente une lunette pour rejoindre l'astre de la nuit. Cyrano de Bergerac, je crois, décrit treize manières d'y accéder.

Ce passage secret, même s'il n'est pas fléché, il faudra le parcourir dans les deux sens. Les Égyptiens, qui étaient moins préoccupés par le sommeil que par la mort et la réincarnation, décrivent dans leur «Livre des morts» le chemin que l'âme doit parcourir, mais cette fois pour ne plus avoir à revenir.

Freud nous ramène à des considérations plus prosaïques : «Nous ne sommes pas habitués à beaucoup méditer sur le fait que l'homme chaque nuit dépouille les enveloppes dont il a recouvert sa peau et aussi éventuellement les accessoires qu'il utilise pour compléter ses déficiences par un substitut : lunettes, cheveux postiches, fausses dents, etc. On peut ajouter qu'en allant se coucher, il dévêt de façon tout à fait analogue, son psychisme, renonçant à la plupart de ses acquisitions psychiques, de sorte que, des deux côtés, il se rapproche à l'extrême de la situation qui fut le point de départ de son développement » [1].

Dépouillement donc du corps, du personnage social, de l'altérité, du Moi. Retour vers un narcissisme primitif et vers le stade de la satisfaction hallucinatoire du désir.

Freud ne s'intéressera pas plus que cela au moment de l'endormissement. Sa grande affaire c'est le rêve, et, entre le rêve et le sommeil, il n'y a pas d'autre point de contact, d'autres objets communs que les restes diurnes.

Sans négliger cette régression mentionnée par Freud, on pourrait mettre l'accent sur d'autres facettes du problème. 
Cette zone de l'endormissement est à la fois un temps et un espace, elle se parcourt dynamiquement. Mais ce parcours est paradoxal puisqu'il consiste à créer dynamiquement de l'atonie (mort apparente) et du vide (perte de la conscience, de la mémoire...).

Les angoisses que procurent l'administration de drogues narcotiques (anesthésie pour opération chirurgicale) disent bien que dans le sommeil quotidien, il y a une adhésion du dormeur à son état. De même que les difficultés d'endormissement soulignent l'impossible constitution de l'absence.

Atteindre le sommeil, c'est réussir une paralysie de la pensée, la création d'une tête vide, la mise en place d'une activité mentale nulle, d'une mémoire absente. Le corps aussi dans ses capacités perceptives et motrices est négativé.

Avant que le rêve n'exerce sa fonction de gardien du sommeil, il faudra donc traverser cette zone de l'endormissement, créer ce vide dans et par la régression. Cette régression massive, jusque dans les zones archaïques de notre expérience, est marquée d'ambivalences complexes et celles-ci limitent la prime de plaisir régressif et fusionnel que procure l'endormissement. Le corps tout d'abord se met en état d'aperception et le dormeur (le zonard de l'endormissement) perd très vite ses repères cénesthésiques. Du coup, l'espace n'est plus orienté, la flèche du temps vacille. Remarquons que nous ne pouvons décrire tout cela que par la négative.

Les fantaisies de l'endormissement décrivent des perceptions molles, envahissantes, où le sujet et le monde qui l'entoure finissent par se confondre. Les auteurs ayant travaillé sur ce thème utilisent volontiers l'image du sein de la mère se rapprochant de la bouche de l'enfant, insistant sur cette fusion première.

Soulignons, en nous attardant sur cette régression et cette image du sein et de l'enfant, qu'à cette période de sa vie, l'enfant en tant qu'être psychique structuré comme ses parents, en tant que sujet, n'est que potentiel. Il faudra du temps, beaucoup de soins, un bain langagier intense pour qu'une subjectivité se constitue dans une culture. Une fois cette subjectivité constituée, une fois cette potentialité réalisée, il n'y a pas en général de retour en arrière. Selon une formule amérindienne parlant de la naissance d'un enfant : « une voix a trouvé un siège ».

Pourtant, cette régression à l'œuvre dans l'endormissement nous donne une illustration de la tentative, non plus de réaliser la potentialité de la subjectivation, mais la potentialité de l'absence. Il ne s'agit pas ici d'établir une symétrie ou une quelconque réciprocité. Mais, tout de même, ce vide auquel le dormeur tente de se confondre ressemble bien au psychisme en négatif. La mort apparente qu'est le sommeil, représente ce négatif du psychisme, elle en est en quelque sorte le signifiant.

Une fois encore les noctambules guident nos pas dans la nuit. Leur vagabondage de lieu en lieu, où ils tentent vainement de se perdre afin de se dépouiller de leur image sociale, de leurs oripeaux moïques, de ce faux double qui les embarrasse et les empêche de se déprimer afin de ré-engendrer la vérité d'un soi, d'un soi suffisamment narcissique, ce vagabondage n'a que l'apparence d'un abandon du logis. Au matin, le noctambule rentre toujours à la maison parce qu'à vrai dire il ne l'a jamais quittée.

Cette zone de l'endormissement est tout à la fois abandon de soi et recentrage sur l'essentiel d'une subjectivité. C'est probablement pour induire et aider à cet équilibre que la mère chante des berceuses. La mère chante et permet à l'enfant de créer l'illusion d'une présence là où il en éprouvait le besoin. La mère chante et l'entraîne vers la solitude créant la désillusion qui permet l'émergence d'une subjectivité (singulière dirait-on en manière de pléonasme) et sa réalité. La mère chante et prononce les mots qui formeront les restes diurnes, véritables briques du rêve.

Mais, dans les fantaisies de l'endormissement, il est difficile de repérer des «mots», des « paroles ». Pour être rigoureux, il faudrait tenir compte d'une gradation dans cet endormissement. $\mathrm{Si}$ au début les fantaisies produites sont des jeux de rôle avec des personnages qui s'expriment, assez vite les mots s'estompent au profit de représentations de mouvements, de rythmes et de sensations. Le dormeur occupe toutes les places, et les enchainements deviennent confus. Il n'est que d'avoir un mot, une phrase en tête, pour ne plus pouvoir trouver le sommeil.

Ces fantaisies de l'endormissement seraient en quelque sorte entre le vide et les mots, comme elles étaient tout à l'heure entre le vide et la subjectivation, ainsi qu'entre le vide et la création du monde.

Cet entre-deux n'est pas localisable. Les fantaisies de l'endormissement ne viennent pas prendre place dans un espace psychologique déterminé à l'avance. Cet espace intérieur, elles l'engendrent et le sujet est appelé à l'occuper en se construisant.

Le Professeur Jouvet, spécialiste du sommeil, émet l'hypothèse que le rêve sert à ré-engrammer les réflexes et les instincts du dormeur, humain ou animal. Cette zone de l'endormissement offrirait au sujet l'occasion, chaque soir renouvelée, de ré-établir sa base subjective. 
Cette zone de l'endormissement se définirait alors « comme temps constitutif d'un espace intérieur » (Fedida) où les restes diurnes, les mots, seraient dévalués, désactivés au rythme du dépouillement des artifices du Moi.

Heureusement, le sommeil n'est qu'une mort apparente et le Moi garde un ail ouvert. Heureusement et malheureusement, car il faudra alors au sommeil un gardien et les restes des restes diurnes serviront au sujet à dire ses désirs avant de s'en débarrasser.

Entre vie et mort, entre éveil et sommeil, cet espace-temps est transitionnel au sens populaire du terme, mais aussi au sens winnicottien : « transaction hallucinatoire ou pré-hallucinatoire qui répond à la fonction économique d'une pré-interprétation du rêve ».

Le dormeur doit croire en la maitrise de ses pulsions et en son contrôle de la régression afin d'aménager une aire d'illusion (ou transitionnelle) aconflictuelle. Cette aire d'illusion est chargée de remplacer la mère et sa capacité d'apaisement.

Winnicott, tentant de représenter schématiquement l'aire transitionnelle, dessine la forme d'un oreiller. Les spectacles auxquels nous nous rendons le soir nous aident aussi à créer cette aire d'illusion (Winnicott, toujours lui, a bien montré le fondement des objets culturels). Le spectacle crée une aire d'illusion, un conte à dormir debout, qui nous donne le sentiment de maîtriser les questions qui y sont abordées et dont la principale est bien sûr l'amour. Le spectacle favorise-t-il ou remplace-t-il la relation sexuelle ? Chacun en jugera par lui-même.

Ces moments de spectacles, ces places où nous nous rassemblons fonctionnent comme des aires d'illusion intermédiaires localisables spatialement et temporellement. Elles dureront l'espace d'une soirée, le temps nécessaire à la restauration narcissique aconflictuelle. Elles nous aideront sans doute à bien dormir, à rêver peut-être.

Sleeping... Dreaming may be

\section{RÉFÉRENCE BIBLIOGRAPHIQUE}

1. Freud S. Complément métapsychologique à la théorie du rêve. In: Métapsychologie. Paris; Gallimard, 1968.

\title{
Communiqué
}

\section{ASM 13 \\ XXXIX Journée de Travail du Centre Alfred Binet}

\section{L'enfant déprimé}

\author{
Président : \\ Docteur Augustin Jeanneau \\ Psychiatre, Psychanalyste, Membre titulaire de la SPP, \\ Ancien Directeur général de l'ASM13 \\ Avec la participation du docteur Paul Denis \\ Psychiatre, Psychanalyste, Membre titulaire de la SPP
}

Samedi 3 décembre 2005

$9 \mathrm{~h}-18 \mathrm{~h}$

Union Internationale des Chemins de Fer, 16, rue Jean Rey, 75015 Paris, France

\author{
Renseignements \\ ASM13, 76, avenue Edison, 75013 Paris, France \\ Tél : 0140774340 -Fax : 0140774355 \\ Email : asm13@asm13.org \\ Site : www.asm 13.org
}

